\title{
PROTÓTIPO DE UM SISTEMA PARA SUPERVISÃO E CONTROLE DO FLUIDO DE PERFURAÇÃO EM PLATAFORMAS ONSHORE
}

\section{PROTOTYPE OF A SYSTEM FOR SUPERVISION AND CONTROL OF ONSHORE PLATFORMS DRILLING FLUID}

\section{S. F. LUAN VICTOR ${ }^{1 *}$, B. C. R. SARA REGINA ${ }^{2}$}

${ }^{1}$ Universidade Federal de Alfenas, Departamento de pós-graduação de Engenharia Química, Poços de Caldas, Minas Gerais, Brasil.

${ }^{2}$ Universidade Estadual da Paraíba, Departamento de Química - Centro de Ciência e Tecnologia, Campina Grande, Paraíba, Brasil.

${ }^{*}$ Corresponding author. Federal University of Alfenas, Department of Chemical Engineering, Poços de Caldas, Minas Gerais, Brazil, Phone: +55 35997374879 e-mail addressl: lvictorbr92@gmail.com (S. F. Luan Victor).

\begin{tabular}{|c|}
\hline ARTICLE INFO \\
\hline $\begin{array}{l}\text { Article history: } \\
\text { Received 2018-10-15 } \\
\text { Accepted 2018-12-20 } \\
\text { Available online 2019-04-25 }\end{array}$ \\
\hline $\begin{array}{l}\text { palavras-chave } \\
\text { Circuitos Elétricos } \\
\text { Planta Didática } \\
\text { Processo } \\
\text { Software }\end{array}$ \\
\hline $\begin{array}{l}\text { keywords} \\
\text { Electrical Circuits } \\
\text { Teaching plant } \\
\text { Process } \\
\text { Software }\end{array}$ \\
\hline
\end{tabular}

\section{A B S T R A C T}

The petroleum industry aims at greater efficiency in drilling wells and reducing costs in this operation. The promotion of a substantial improvement in the logistics used in this process is the challenge to be beaten, so this study aims to explore the method of circulation and separation of the drilling fluid in onshore platforms, creating a didactic plant of this system. It is proposed to elaborate the electric circuits of the pump and the solenoid valves, showing its operation, the construction of the supervision software and the programming of the programmable logic controller (PLC) to switch with the control synoptic; the issues related to supervision and control of and process in a didactic plant are presented, as well as the equipment control and drilling fluid flow. The concepts and solutions addressed in this article are decisive for the use of supervision software and its applicability in the didactic plan, training students in the area of oil and gas, making them aware of the economic and legal aspects of the procedure.

RES UM O/R ES UM EN

A indústria petrolífera visa maior eficiência na perfuração de poços e diminuição dos custos nesta operação. A promoção de uma substancial melhoria na logística utilizada neste processo é o desafio a ser batido, assim, este estudo visa explorar o método de circulação e separação do fluido de perfuração em plataformas onshore, criando uma planta didática deste sistema. Propõe-se a elaboração dos circuitos elétricos da bomba e das válvulas solenoides, mostrando o seu funcionamento, a construção do software de supervisão e a programação do controlador lógico programável (CLP) para comutar com o sinótico de controle; apresentase as questões relacionadas à supervisão e controle deste processo numa planta didática, bem como o controle dos equipamentos e fluxo do fluido de perfuração. Os conceitos e soluções abordados neste artigo são determinantes para a utilização do software de supervisão e sua aplicabilidade na planta didática profissionalizando discentes na área de petróleo e gás, conscientizando-os dos aspectos econômicos e legais referentes ao procedimento. 


\section{INTRODUÇ̃̃̃O}

Nas atividades petrolíferas, a supervisão e controle dos sistemas de circulação e separação devem ser tratados com importância fundamental, pois estes fluidos devem permitir o resfriamento da broca, carregar os cascalhos gerados na perfuração até a superfície (trazendo consigo informações da formação rochosa) e manter a estabilidade do poço (SILVA, 2018). O fluido tem como característica propícia se transformar em uma espécie de gel para conter os cascalhos, mantendo-os em suspensão a qualquer parada da circulação, todavia, tem como principal revés poder incorporar produtos de constituição tóxica, corrosiva ou até mesmo agressivas ao meio ambiente (RADZLAN, 2014).

$\mathrm{O}$ aprendizado na área de processos industriais requer atividades em laboratórios (RODRIGUES, 2018) a fim de que seja simulado o que o profissional verá quando for atuar na sua área, isso é possível por intermédio das plantas didáticas. As plantas didáticas presentes nos laboratórios universitários de engenharia, em sua maioria, possuem limitações, pois suas simulações, se comparadas com a realidade da prática industrial, quase sempre se comporta como se os problemas de engenharia apresentassem soluções ideais.

Nesse viés, o objetivo principal do trabalho, além de desenvolver um estudo levantando informações sobre o sistema de circulação e separação do fluido de perfuração em plataformas onshore, é elaborar uma planta didática sobre este processo que possibilite a implementação de práticas mais próximas da realidade para que possam ser geradas situações de melhorias pela indústria de petróleo, centros de pesquisas, universidades e agencias de controle governamental. Notadamente, realiza-se uma análise do processo de circulação e separação do fluido de perfuração, de forma a supervisionar, controlar e aferir o processo.

Para tanto, foi necessário a elaboração de um software demonstrativo que executará o processo de supervisão e controle de circulação do fluido de perfuração em uma planta didática, bem como a elaboração do seu respectivo programa em ladder (Linguagem de programação) para que este procedimento possa ser ilustrado possibilitando melhoria do processo em projetos futuros.

Essas etapas são essenciais para que protótipo funcione de forma adequada e proporcionem aos alunos e professores uma simulação o mais real possível, melhorando o aprendizado.

\section{MATERIAIS E MÉTODOS}

O trabalho foi desenvolvido na Escola SENAI Antônio Souza Noschese utilizando e configurando com o software SCADA (Supervisory Control and Data Acquisition) InduSoft Web Studio® v6.1, programando a automação com o CLP ATOS modelo 4004.06BF usando a interface de comunicação RS232 em um modelo físico tipo cabo elétrico CRS232415. Preliminarmente, foi realizado uma pesquisa bibliográfica, em seguida, foram aplicados esses conhecimentos na idealização da planta didática. Junto com a problematização da planta, foi iniciada a escrita deste trabalho.

No decorrer da produção desse artigo, foram consultadas publicações indexadas nas principais plataformas da área, tais como a OnePetro ${ }^{\circledR}$ e Journal of Petroleum Technology para aprimorar a ideia sobre o tema sugerido, e fazer com que este supervisório de controle fosse fiel e aplicável. Diante disto, um protótipo do sistema de circulação do fluido de perfuração em plataformas onshore foi elaborado em escala reduzida para facilitar a visualização do processo em ação. Para tanto, foi projetado o esquema elétrico deste modelo com ajuda de softwares de desenho técnico, AutoCAD ${ }^{\circledR}$ versão 2013, conforme Figura 1 e 2.

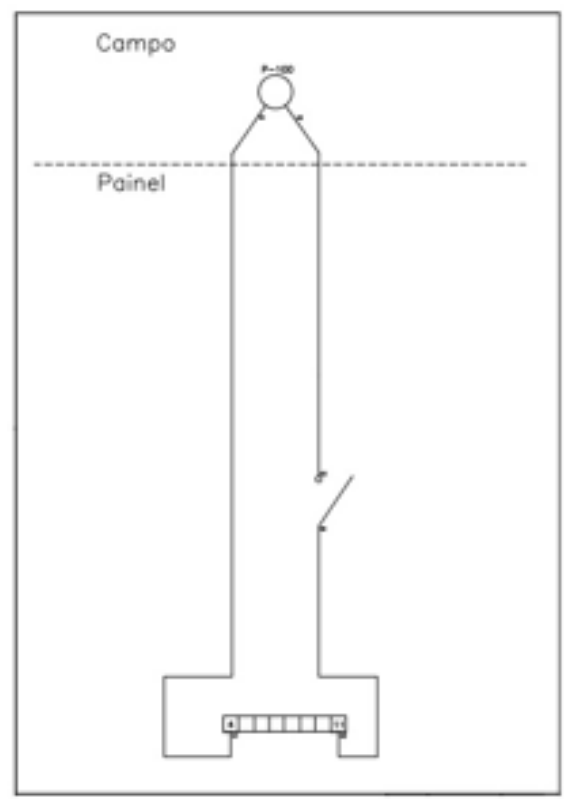

Figure 1 - Esquema elétrico da bomba.

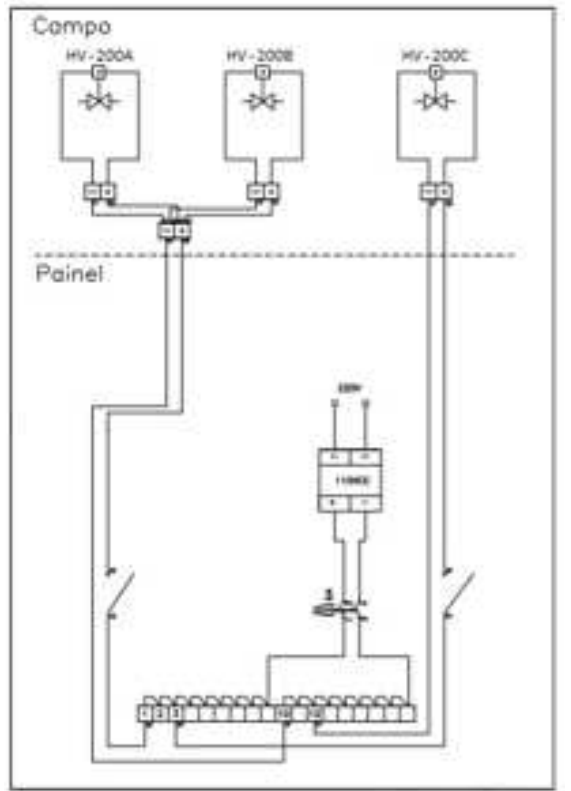

Figure 2 - Esquema elétrico das válvulas.

Após esta etapa de elaboração do escopo do trabalho foi realizada uma investigação cronológica com o intuito de levantar dados pertinentes a área de estudos. Segundo Lüdke e André, 1986, a pesquisa de viés qualitativo ou naturalístico "envolve a obtenção de dados descritivos, obtidos no contato 
direto do pesquisador com a situação estudada, enfatiza o processo do que o produto e se preocupa em retratar a perspectiva dos participantes". Esse tipo de pesquisa pode ser desenvolvida aliando a descrição à explicação de modo a se ter uma visão de totalidade do fenômeno, relacionando a situação em pauta às esferas sociais mais amplas: "trabalhar com a pesquisa qualitativa numa abordagem sócio histórica consiste, numa preocupação de compreender os eventos investigados, descrevendo-os e procurando as suas possíveis relações" (Freitas, 2002, p. 28).

O descritivo operacional da planta didática dividido em duas operações: Partida da bomba ligando o sistema de operação da planta e o acionamento das válvulas solenoides mostrando como deve estar o alinhamento que permite a alimentação dos tanques.

A planta didática tem como finalidade simular um processo industrial, como é utilizado para fins didáticos, o único produto de manuseio pela planta é a água que circula entre três tanques, sendo que esses tanques são abertos. A Figura 3 mostra a planta didática e o fluxo do produto gerados por ela.

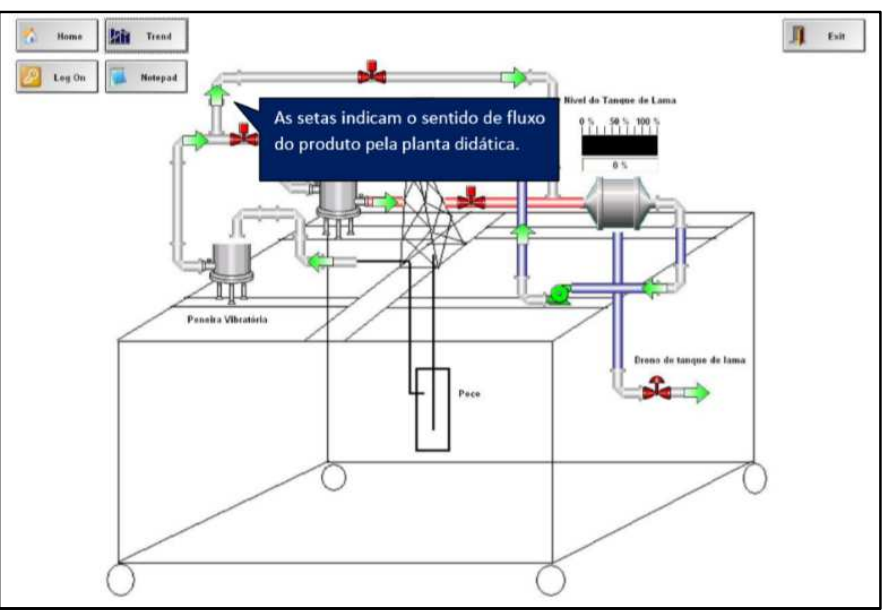

Figure 3 - Apresentação do fluxo da planta didática.

A Figura 4 auxilia na navegação do programa.

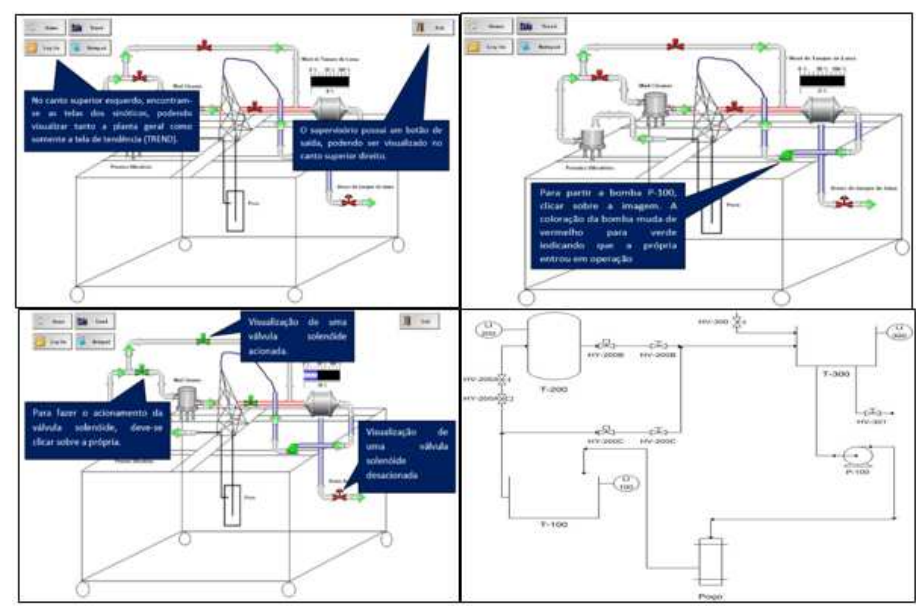

Figura 4 - Sinótico geral do supervisório, bomba P-100 da planta didática, acionamento das válvulas solenoids e fluxograma de processo da planta didática.

Para a integração deste sistema supervisório com a planta didática, foi necessário a utilização de um controlador lógico programável (CLP) Atos modelo 4004.06BF utilizando como recurso para comunicação a interface de RS232 em meio físico tipo cabo elétrico.

Os materiais necessários para este procedimento são o Kit CLP Atos MPC 4004 para fazer todo o controle e comunicação entre a planta física e o supervisório, microcomputador com o software Indusoft ${ }^{\circledR}$ para o controle remoto da planta didática, microcomputador com o software A1 para fazer a programação e integração do IHM (Interação Humano-Máquina) com SCADA e da interface Ethernet/serial RS232/RS485 compatível com o protocolo Mudbus para a leitura e escrita das variáveis e realizar as conecções elétricas do sensor ao transmissor e ao aquisitor de dados.

É importante ter em mãos os documentos de leitura: manual do CLP Atos 4004 e manual drive de comunicação Modbus - Indusoft巴.

Os testes de integração do CLP com o supervisório foram feitos na Escola SENAI Antonio Souza Noschese.

A Figura 5 a seguir mostra a programação feita no software A1 para que este possa ser baixado no CLP da Atos a fim de estabelecer a comunicação com o supervisório de controle.

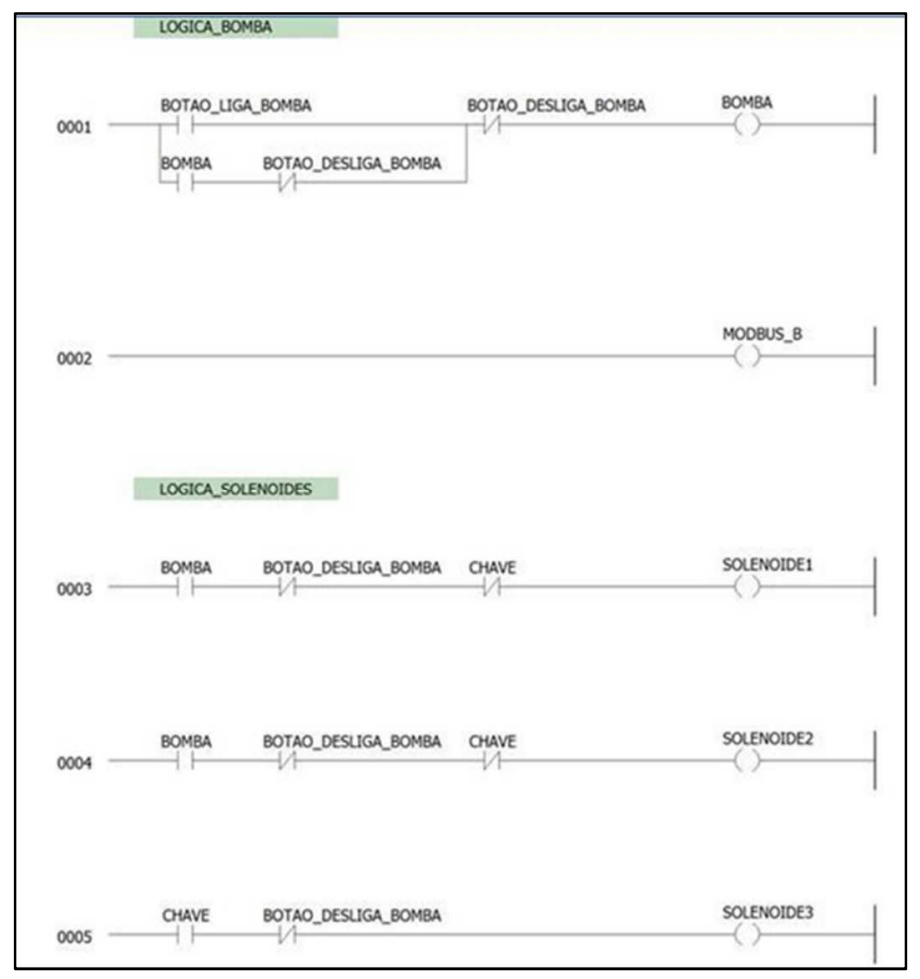

Figura 5 - Programação feita em Ladder.

Os endereços dos pontos discretos de I/O (Entrada e Saída) do CLP utilizados seguem como na Tabela 1 a seguir, em que o tipo de dado é descrito como booleano (BOOL), sendo que os valores iniciais dos endereços de entrada (\% I0.0 - \% I0.7) podem ser zero (0) ou um (1) e os valores iniciais dos endereços de saída (\%Q0.0 - \%Q0.7) são considerados como falso, ou seja, seu valor de saída é zero (0). 
Table 1 - Quadro de variáveis globais

\begin{tabular}{|c|c|c|c|c|c|c|}
\hline Endereço & Nome & Tipo de dado & $\begin{array}{l}\text { Valor } \\
\text { inicial }\end{array}$ & Descrição & $\begin{array}{c}\text { Endereço } \\
\text { Físico }\end{array}$ & $\begin{array}{l}\text { Posição } \\
\text { Modbus }\end{array}$ \\
\hline$\% \mathrm{I} 0.0$ & BOTÃO_LIGA_BOMBA & BOOL & - & Digital Input 0 & 0000 & 1 \\
\hline$\% \mathrm{I} 0.1$ & BOTÃO_DESLIGA_BOMBA & BOOL & - & Digital Input 1 & 0001 & 2 \\
\hline$\% \mathrm{I} 0.2$ & SOLENOIDE1 & BOOL & - & Digital Input 2 & 0002 & 3 \\
\hline$\% \mathrm{I} 0.3$ & SOLENOIDE2 & BOOL & - & Digital Input 3 & 0003 & 4 \\
\hline$\% \mathrm{I} 0.4$ & SOLENOIDE3 & BOOL & - & Digital Input 4 & 0004 & 5 \\
\hline$\% \mathrm{I} 0.5$ & CHAVE & BOOL & - & Digital Input 5 & 0005 & 6 \\
\hline$\% \mathrm{I} 0.6$ & - & BOOL & - & Digital Input 6 & 0006 & 7 \\
\hline$\% \mathrm{I} 0.7$ & - & BOOL & - & Digital Input 7 & 0007 & 8 \\
\hline$\% \mathrm{Q} 0.0$ & LAMPADA1 & BOOL & FALSE & Digital output 0 & 0200 & 3001 \\
\hline$\%$ Q0.1 & LAMPADA2 & BOOL & FALSE & Digital output 1 & 0201 & 3002 \\
\hline$\% \mathrm{Q} 0.2$ & BOMBA & BOOL & FALSE & Digital output 2 & 0202 & 3003 \\
\hline$\% \mathrm{Q} 0.3$ & - & BOOL & FALSE & Digital output 3 & 0203 & 3004 \\
\hline$\% \mathrm{Q} 0.4$ & - & BOOL & FALSE & Digital output 4 & 0204 & 3005 \\
\hline$\%$ Q 0.5 & - & BOOL & FALSE & Digital output 5 & 0205 & 3006 \\
\hline$\% \mathrm{Q} 0.6$ & - & BOOL & FALSE & Digital output 6 & 0206 & 3007 \\
\hline$\% \mathrm{Q} 0.7$ & - & BOOL & FALSE & Digital output 7 & 0207 & 3008 \\
\hline
\end{tabular}

\section{RESULTADOS E DISCUSSÕES}

Os fluidos de perfuração são vistos de várias maneiras por diferentes autores. De modo geral, o fluido de perfuração é qualquer fluido circulante capaz de tornar a operação de perfuração viável (Thomas et al., 2002). Apesar disso, autores como Thomas et al., 2002) analisam os fluidos de perfuração como misturas complexas de sólidos, líquidos, produtos químicos e, por vezes, até gases. Sendo que, do ponto de vista químico, eles podem assumir aspectos de suspensão, dispersão coloidal ou emulsão, dependendo do estado físico dos componentes. Do ponto de vista físico, os fluidos de perfuração tendem a assumir o mesmo comportamento de fluidos nãonewtonianos, ou seja, possui a relação entre a taxa de cisalhamento e a taxa de deformação não é constante (MACHADO, 2002, p.26).

A construção de um poço inclui várias etapas de segurança e notadamente, de limpeza, pois ao realizar o procedimento de perfuração, o fluido retirado vem contaminado por uma série de elementos. Assim, entra em função o sistema de circulação de fluidos da sonda, que é responsável pelo bombeamento dos fluidos de perfuração e da pasta de cimento para o poço; cuja função será carregar o cascalho para fora do poço, conferir pressão hidrostática ao poço, lubrificar e refrigerar a broca, bem como depositar uma camada de reboco na parede do poço (ISRAEL et. al., 2018).

Os sistemas supervisórios dão margem para que sejam monitoradas e rastreadas informações de um processo ou instalação física. Tais informações são coletadas através de equipamentos de aquisição de dados e, em seguida, manipuladas, analisadas, armazenadas e posteriormente, apresentadas ao usuário. Estes sistemas também são chamados de SCADA.

Atualmente, os sistemas de automação industrial utilizam artifícios de computacionais e redes de comunicação IHM para otimizar o monitoramento e controle dos processos, podendo, com isso, coletar os dados em locais de difícil acesso, que são posicionados de forma dispersa geograficamente, e apresenta uma plataforma mais acessível para o operador, com recursos gráficos e conteúdo multimídia (CAVALIERI, 2013).

Nos últimos anos, para administrar processos industriais, torna-se necessária a posse de conhecimentos sobre os materiais ou fluidos de entrada e saída do processo e um bom monitoramento do procedimento. O controle de uma planta é realizado por meio de transmissores instalados ao longo do processo para que, caso haja alguma alteração durante o procedimento, ainda em tempo hábil, atuará na entrada mantendo assim o processo estável. Há muitas formas e diferentes tipos de ações que facilitam a execução do controle de um processo e, um dos meios mais facilitadores para o controle do processo, são os supervisórios de monitoração (CARVALHO, 2018, p.10).

O supervisório do processo foi desenvolvido para facilitar o controle e garantir, ainda mais, a segurança do sistema em que foi destinado a monitorar, construindo uma melhora significativa no controle do processo (MUNSHI et. al., 2017). A implementação do supervisório do processo foi feita em Controlador Lógico Programável (CLP), através da linguagem ladder. Essa é uma linguagem de programação de baixo nível e trata-se de um auxílio gráfico para a programação de CLPs, que possibilita o controle de sistemas críticos e/ou industriais. A vantagem da representação das lógicas de controle em diagramas ladder é que os engenheiros e técnicos de campo podem desenvolver o software sem conhecer previamente outras lógicas de programação.

A criação de um protótipo do sistema de circulação do fluido de perfuração em plataformas onshore em tamanho reduzido tornou possível ilustrar o processo em geral, para que este possa ser melhor visto, e, dessa foram, que o mesmo possa ser a base para projetos futuros. Dessarte, o mesmo pode ser considerado uma ferramenta importante didaticamente, visto que permite o aprendizado por meio da realização de uma análise do processo de circulação e separação do fluido de perfuração feita em base na simulação dessa planta didática, de forma a supervisionar, controlar e aferir o processo. 


\section{CONCLUSÕES}

Adentrar e enriquecer o conhecimento acerca do sistema de circulação do fluido de perfuração e trazer à tona não somente o sistema de circulação do fluido em si, como também possíveis processos que contaminam o meio ambiente.

Este estudo tentou transmitir informações que possibilita o entendimento do processo de circulação do fluido de perfuração, bem como transmitir também uma aprimoração na área de supervisão e controle das unidades de circulação do fluido de perfuração para que haja mais segurança no processo e uma redução em possíveis contaminações ou, no mínimo, a redução deste tipo de contaminação aos parâmetros aceitáveis na legislação, o que foi possível através das simulações feitas com o protótipo.

Também o enfoque da elaboração da planta didática com o processo de supervisão e controle do sistema de circulação do fluido de perfuração em plataformas onshore (plataformas terrestres), visa ainda uma abordagem sobre melhorar a capacitação de profissionais que possuem interesse em entender melhor como essa tecnologia funciona ou curiosos que possuem interesses sobre o assunto. Considerando que não há quase nenhuma divulgação sobre o controle e supervisão desse processo, é necessário que haja melhor material de estudo, como a planta didática e do software desenvolvidos e implementados como o descrito no desenvolvimento do presente estudo.

Os testes de integração do CLP do supervisório foram feitos na Escola SENAI Antonio Souza Noschese, comprovando a funcionalidade da planta e do software de supervisão. Com a finalização da estrutura física e do software de monitoração e controle do processo da Planta Didática, conclui-se a importância da mesma para seu caráter didático e tecnológico.

Essa aplicação pode ser realizada tanto em laboratórios para o aprendizado de discentes durante a graduação em cursos de engenharia, quanto em aplicações práticas em cursos de capacitação profissional, contribuindo dessa forma tanto para formação acadêmica, quanto para os meios de serviço, por conseguinte, fica evidente a importância desse estudo para a formação curricular e empírica dos profissionais de campo e alunos a posteriori.

\section{R E F E R E N C I A S}

CARVALHO, Eduardo Felipe Gomes de. Dimensionamento, automação, aquisição de dados e controle supervisório de um trocador de calor do tipo casco e tubo. 2018. 38f. Trabalho de Conclusão de Curso (Graduação em Engenharia Química) - Departamento de Engenharia Química, Universidade Federal do Rio Grande do Norte, Natal, 2018.

CAVALIERI, Salvatore; CHIACCHIO, Ferdinando. Analysis of OPC UA performances. Computer Standards \& Interfaces, v. 36, n. 1, p. 165-177, 2013.

FREITAS, M. T. de A. A abordagem sócio-histórica como orientadora da pesquisa qualitativa. In: Cadernos de Pesquisa (Fundação Carlos Chagas), São Paulo, v. 1, n. 116, p. 21-40, 2002.

GROENNER BARBOSA, Bruno. Introdução à Engenharia de Controle e Automação [Apresentação em PowerPoint].
Lavras; Universidade Federal de Lavras, 2006.

ISRAEL, R., MCCRAE, D., SPERRY, N., GORHAM, B., THOMPSON, J., RAESE, K., ... COIT, A. (2018). Delivering Drilling Automation II - Novel Automation Platform and Wired Drill Pipe Deployed on Arctic Drilling Operations. Society of Petroleum Engineers. doi:10.2118/191574-MS

LÜDKE, Menga e ANDRÉ, Marli E. De. Pesquisa em Educação: Abordagens qualitativas. São Paulo: EPU, 1986.

MACHADO, J. C. Fundamentos e Classificação de Fluidos Viscosos. Reologia e Escoamento de Fluidos-Ênfase na indústria do petróleo. Editora Interciência. Rio de Janeiro, 2002. Páginas 1-40.

MUNSHI, A. A., O'CONNOR, K., MCMAHON, S., \& CARMODY, M. (2017). Deployment of a Remotely Activated Liner Hanger System to Improve Drilling and Well Construction Efficiencies. Society of Petroleum Engineers. doi:10.2118/187108-MS

RADZLAN, R.; YUSUP, S.; YUSUF, N. R.; KAMIL, R. N. M.; $\mathrm{CHOK}, \mathrm{C}$. The effect of lime and primary emulsifier on the rheological behaviour of palm fatty acid distillate (pfad) biodiesel - based drilling fluid. Australian Journal of Basic and Applied Sciences. Thailand, v. 5, p.267-272. 25 abr. 2014. Disponível em: <http://ajbasweb.com/old/ajbas/2014/Special\%202/267272.pdf>. Acesso em: 11 outubro 2018.

RODRIGUES, Bernardo Oricchio. Ciências, agora no laboratório: as ideias pedagógicas de John Dewey aplicadas em um laboratório de ciências. 2018. xii, 101 f., il. Dissertação (Mestrado Profissionalizante em Ensino de Ciências) - Universidade de Brasília, Brasília, 2018.

SILVA, Samara Leandro. Estudo da Atapulgita como viscosificante para fluido de perfuração aquoso salgado. 2018. 48f. Trabalho de Conclusão de Curso (Graduação) - Curso de Engenharia de Petróleo, Universidade Federal do Rio Grande do Norte, Natal, Brasil, 2018.

THOMAS, J. E. (Org). Fundamentos de Engenharia do Petróleo. Editora Interciência. Rio de Janeiro, 2002. Páginas 81-87. 